\title{
Suppression of lung cancer metastasis-related protein 1 promotes apoptosis in lung cancer cells
}

\author{
YANG XU, CHUNSUN LI, QING TIAN, YANQIN LI, ZHEN YANG, ZHIXIN LIANG and LIANGAN CHEN \\ Department of Respiratory Diseases, Chinese PLA General Hospital, Beijing 100853, P.R. China
}

Received July 15, 2012; Accepted September 14, 2012

DOI: $10.3892 /$ ijmm.2012.1160

\begin{abstract}
Lung cancer is one of the most common human cancers and the leading cause of cancer-related death worldwide. The identification of lung cancer associated genes is essential for the diagnosis and treatment of this disease. Lung cancer metastasis-related protein 1 (LCMR1) is a novel gene cloned by differential display polymerase chain reaction in our laboratory. This study was designed to investigate the role of LCMR1 in the generation of apoptosis in lung cancer cells. RNA interference (RNAi) approach was used to knock down LCMR1 in lung cancer cells. The impacts of LCMR1 knockdown on the apoptosis of lung cancer cells were assessed using flow cytometry, quantitative real-time RT-PCR and western blotting. The results demonstrated that the inhibition of LCMR1 by RNAi enhanced apoptotic activity in lung cancer cells and this process required the involvement of p53. In addition, LCMR1 knockdown resulted in the upregulation of Bax and downregulation of Mcl-1. In conclusion, LCMR1 suppresses apoptosis in lung cancer cells and this effect is associated with multiple apoptosis-related proteins, including p53, Bax and Mcl-1. These results provide new insights into the potential role of LCMR1 in the regulation of lung cancer carcinogenesis.
\end{abstract}

\section{Introduction}

Lung cancer is one of the most common human cancers worldwide (1). Despite continuous improvement in treatments,

Correspondence to: Professor Liangan Chen, Department of Respiratory Diseases, Chinese PLA General Hospital, 28 Fuxing Road, Beijing 100853, P.R. China

E-mail: chenliangan301@163.com

Abbreviations: LCMR1, lung cancer metastasis-related protein 1; RNAi, RNA interference; NCBI, National Center for Biotechnology Information; ORF, open reading frame; Med19, mediator complex subunit 19; NSCLC, non-small cell lung cancer; RPMI-1640, Roswell Park Memorial Institute medium 1640; DMEM, Dulbecco's modified Eagle's medium; siRNA, small interfering RNA; TBST, Tris-buffered saline Tween-20; PI, propidium iodide; Bcl-2, B-cell lymphoma 2

Key words: lung cancer metastasis-related protein 1, RNA interference, lung cancer, apoptosis lung cancer remains the main cause of cancer-related deaths (2). Therefore, it is essential to understand the molecular mechanisms of this carcinogenesis, so that effective therapeutic strategies may be developed.

Emerging evidence has shown that tumorigenesis is a complex multi-step process associated with genetic alterations (3). Identification of genes that are involved in cancer development is essential for lung cancer diagnosis and treatment.

Lung cancer metastasis-related protein 1 (LCMR1) is a novel gene cloned from PLA-801, a poorly differentiated human large-cell lung carcinoma cell line, using a differential display polymerase chain reaction technique in our laboratory in 2002 (4). Information regarding this gene has been submitted to the National Center for Biotechnology Information (NCBI), and a Genbank accession number (AY148462) has been assigned. LCMR1 is located on human 11q12.1 chromosome locus and is comprised of 949 nucleotides with an open reading frame (ORF) encoding for a peptide with 177 amino acids. In the human genome, LCMR1 is also known as the mediator complex subunit 19 (Med19). Med19 is a component of the mediator complex, which is a coactivator for DNA-binding factors that activates a transcription via RNA polymerase II (5).

The distribution of LCMR1 has been demonstrated as site-dependent (4). A high level of LCMR1 expression is present in the heart, skeletal muscle, kidney, liver and placental tissues. However, the expression levels are low in the brain, colon, thymus, spleen, small intestine, lung and peripheral blood leukocytes. To explore the relation between LCMR1 and lung cancer, we previously examined LCMR1 expression in 84 human non-small cell lung cancer (NSCLC) tissues using immunohistochemistry. LCMR1 was overexpressed in NSCLC and its expression was significantly associated with the clinical stage of the disease. These results suggest that LCMR1 plays a critical role in the oncogenesis of lung cancer (4).

Recent studies have reported that LCMR1 is implicated in several important cellular processes including cell proliferation, cell cycle and oncogenesis of cancer (6-8). However, the function of LCMR1 in the regulation of apoptosis remains unclear. In the present study, we employed RNA interference (RNAi) technique to knock down LCMR1 expression and we investigated the regulatory role of LCMR1 in the generation of lung cancer cell apoptosis. Our results demonstrated that LCMR1 participated in the regulation of the apoptosis of lung cancer cells. This effect was p53-dependent and was 
associated with Bax and Mcl-1. These findings suggest that LCMR1 may serve as a potential molecular target for lung cancer therapies.

\section{Materials and methods}

Cell lines and culture. The 95D cell line, subcloned from a poorly differentiated human large-cell lung carcinoma cell line PLA-801, was kindly provided by Dr Lezhen Chen (Department of Pathology, Chinese PLA General Hospital, China). A549 and H1299 cell lines were a gift from Professor ZhiHua Liu (Chinese Academy of Medical Sciences and Peking Union Medical College, China). 95D cells were cultured in Roswell Park Memorial institute medium RPMI1640. A549 and H1299 cells were cultured in Dulbecco's modified Eagle's medium (DMEM). Both media were supplemented with $10 \%$ fetal bovine serum, penicillin $(100 \mu \mathrm{g} / \mathrm{ml})$ and streptomycin $(100 \mu \mathrm{g} / \mathrm{ml})$. The cells were maintained in an incubator with a humidified atmosphere of $5 \% \mathrm{CO}_{2}$ at $37^{\circ} \mathrm{C}$.

Small interfering RNA and cell infection. The human LCMR1-specific small interfering RNA (siRNA) sequence, 5'-GAGAGAGAGGGACAUGCUU-3', was designed using an online siRNA tool provided by Invitrogen Life Technologies (USA) with the LCMR1 sequence (GenBank code: AY148462) as a reference. The negative control (NC) sequence was 5'-CCUACGCCACCAAUUUCGU-3'. For each cell line, there were 3 experimental groups: cells without infection (CON group), cells infected with the negative control siRNA (NC group) and cells infected with the LCMR1-siRNA (RNAi group). Cells were seeded in a 6-well plates and transfected at $\sim 30-40 \%$ confluency using Lipofectamine 2000 (Invitrogen) according to the manufacturer's protocol.

Quantitative real-time PCR. Total RNA was extracted from the cells using Trizol reagent (Invitrogen Life Technologies) and was reverse transcribed using MMLV Reverse Transcriptase (Promega Corporation, Madison, WI, USA) according to the manufacturer's protocol. Quantitative real-time PCR reactions were prepared in a volume of $20 \mu \mathrm{l}$ containing $0.5 \mu \mathrm{l}$ cDNA sample, $0.5 \mu \mathrm{l}$ $10 \mu \mathrm{M}$ primers, $10 \mu \mathrm{l} 2 \mathrm{X}$ SYBR Premix Ex Taq (Takara,

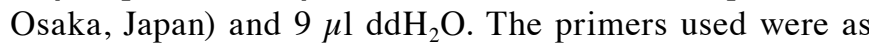
follows: $\beta$-actin, 5'-CATGTACGTTGCTATCCAGGC-3' (forward) and 5'-CTCCTTAATGTCACGCACGAT-3' (reverse); LCMR1, 5'-AACAGAGCCGTACCCAGGAT-3' (forward) and 5'-GGGTGGTCTGGACATTGTC-3' (reverse); Bax, 5'-TGGAGCTGCAGAGGATGATTG-3' (forward) and 5'-GAAGTTGCCGTCAGAAAACATG-3' (reverse); Mcl-1, 5'-CTCATTTCTTTTGGTGCCTTT-3' (forward) and 5'-CCAGTCCCGTTTTGTCCTTAC-3' (reverse). Quantitative real-time RT-PCR analysis was performed on MyiQ2 (BioRad, USA). The relative quantity of mRNA was calculated using the $2^{-\Delta \Delta \mathrm{CT}}$ method with the $\beta$-actin mRNA level as the reference for normalization. All experiments were repeated at least 3 times.

Protein extraction. The total protein of the cells was extracted by addition of a lysis buffer $(50 \mathrm{mM}$ Tris $\mathrm{pH} 7.6,10 \mathrm{mM}$ EDTA, $150 \mathrm{mM} \mathrm{NaCl}, 0.1 \%$ NP-40, 2 mM dithiothreitol,
$1 \mathrm{mM}$ phenylmethylsulfonyl fluoride, $0.7 \mu \mathrm{g} / \mathrm{ml}$ pepstatin $\mathrm{A}$, $10 \mu \mathrm{g} / \mathrm{ml}$ leupeptin and $1 \mu \mathrm{g} / \mathrm{ml}$ aprotinin) for $50 \mathrm{~min}$ at $4^{\circ} \mathrm{C}$. The lysates were then centrifuged at $12,000 \mathrm{rpm}$ for $30 \mathrm{~min}$ at $4^{\circ} \mathrm{C}$. The soluble protein concentrations in the lysates were determined using a BCA Protein Assay kit (Pierce Biotechnology, Inc., Rockford, IL, USA).

Western blotting. For western blot analyses, a 25-50 $\mu \mathrm{g}$ of total protein was separated on a $12 \%$ SDS-PAGE gel and was transferred onto a PVDF membrane. The membrane was blocked in $5 \%$ dried milk for $1 \mathrm{~h}$ at room temperature and was then incubated with a specific primary antibody at $4^{\circ} \mathrm{C}$ overnight. The membrane was then washed three times with Tris-buffered saline Tween-20 (TBST), followed by incubation with a horseradish peroxidase-conjugated secondary antibody at room temperature for $1 \mathrm{~h}$. Following another round of washing with TBST, the membrane was developed using enhanced chemiluminescence (ECL) (Amersham Life Sciences, Amersham, UK). The staining intensity of the bands was quantitated by densitometry, using image analyzing software (Multi Gauge Ver 3.2; Japan).

The antibodies used were as follows: the primary antibodies for $\beta$-actin (Santa Cruz Biotechnology, Inc., USA), LCMR1/ MED19 (Abcam, UK), caspase-3 (New England Biolabs, USA), Bax (Abcam, USA) and Mcl-1 (Santa Cruz Biotechnology, Inc.) were employed at dilutions of 1:500, 1:1000, 1:1000, 1:500 and 1:500, respectively. The secondary antibodies were utilized at working concentrations of 1:5000, 1:5000 and 1:6000 for antigoat, anti-rabbit and anti-mouse IgG (all were from Santa Cruz Biotechnology, Inc.), respectively.

Flow cytometric analysis. Cells were harvested $72 \mathrm{~h}$ after infection by centrifugation at $1,000 \mathrm{rpm}$ for $5 \mathrm{~min}$ and fixed in $70 \%$ cold ethanol overnight. The next day, cells were centrifuged at 1,000 rpm for $5 \mathrm{~min}$, resuspended in PBS, then filtered through a 400-mesh membrane, stained with propidium iodide (PI) in the dark at $4^{\circ} \mathrm{C}$ for $30 \mathrm{~min}$ and analyzed using flow cytometry. All experiments were conducted in triplicate.

Statistical analysis. SPSS 13.0 statistical package was utilized for statistical analysis. The data are expressed as the means \pm SD. Significance was calculated using a Student's t-test. A P-value $<0.05$ was considered to indicate a statistically significant difference.

\section{Results}

Efficacy of RNAi for LCMRl knockdown. To determine the effectiveness of the RNAi for silencing the LCMR1 expression in 95D cells, we examined the changes in the expression levels of LCMR1 3 days after the infection. As displayed in Fig. 1A, the transcriptional expression level of LCMR1, evaluated by qRT-PCR, in the 95D-RNAi cells was significantly lower compared to levels in the parent 95D and 95D-NC cells $(\mathrm{P}<0.05)$. The protein expression level of LCMR1, assessed by western blotting, was also decreased in the 95D-RNAi cells $(\mathrm{P}<0.05)$ (Fig. 1B), which is consistent with the transcriptional change in this gene. These results indicated that the LCMR1siRNA used in the current study effectively reduced the LCMR1 expression at both the mRNA and protein levels. 
A

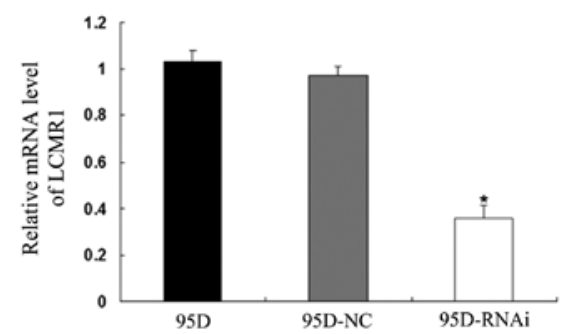

B
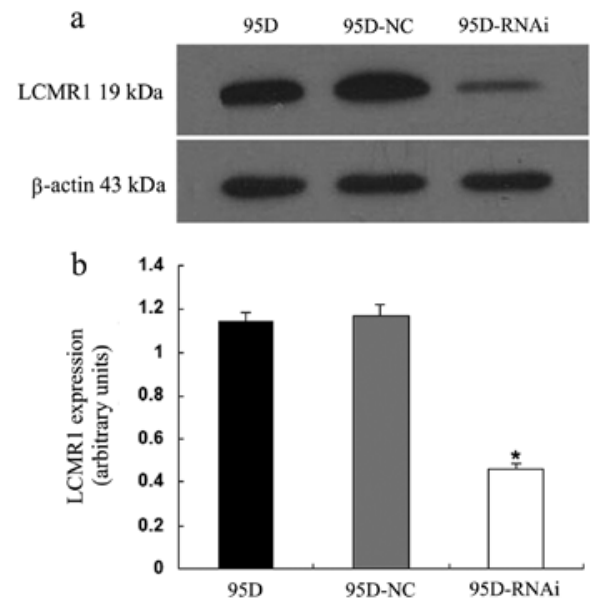

Figure 1. Efficacy of RNAi for LCMR1 knockdown. (A) Comparison of the transcriptional expression levels of LCMR1 in the 95D, 95D-NC and 95D-RNAi cell groups. (B) a, An example of western blot analysis for the LCMR1 protein examined in the 3 cell groups. b, Comparison of the protein expression levels of LCMR1 in the 3 cell groups. The 95D-RNAi cell group displayed a significantly reduced expression level at both the mRNA and protein levels. ${ }^{*} \mathrm{P}<0.05$.

LCMR1 knockdown enhances apoptotic activity. To determine whether LCMR1 knockdown potentiates the apoptotic activity in $95 \mathrm{D}$ cells, we examined the percentages of apoptotic cells in the 95D, 95D-NC and 95D-RNAi cell groups using flow cytometry $72 \mathrm{~h}$ after the infection. As demonstrated in Fig. 2, the percentage of apoptotic cells in the 95D-NC cell group was similar to that in the 95D cell group ( $\mathrm{P}>0.05$ ), whereas the percentage in the 95D-RNAi cell group was significantly higher compared to the 95D and 95D-NC cell groups $(\mathrm{P}<0.05)$. These results suggest that LCMR1 knockdown enhances apoptotic activity in 95D cells.

To further confirm the action of LCMR1 knockdown in apoptosis, we compared the expression levels of cleaved caspase-3, a biological marker of apoptosis, among the three groups using western blot assay. The expression level of cleaved caspase-3 protein in the 95D-RNAi cells was significantly higher when compared to expression levels in the 95D and 95D-NC cells (Fig. 3), suggesting that the suppression of LCMR1 increases the expression of cleaved caspases-3. Collectively, these findings suggest that the downregulation of LCMR1 promotes apoptotic activity in lung cancer cells.

LCMR1-mediated apoptosis is associated with the p53 pathway. Apoptosis has been ascribed as being p53-dependent or p53-independent (9). To determine whether p53 is involved in LCMR1-related apoptosis, we examined the apoptotic activity in two human lung carcinoma
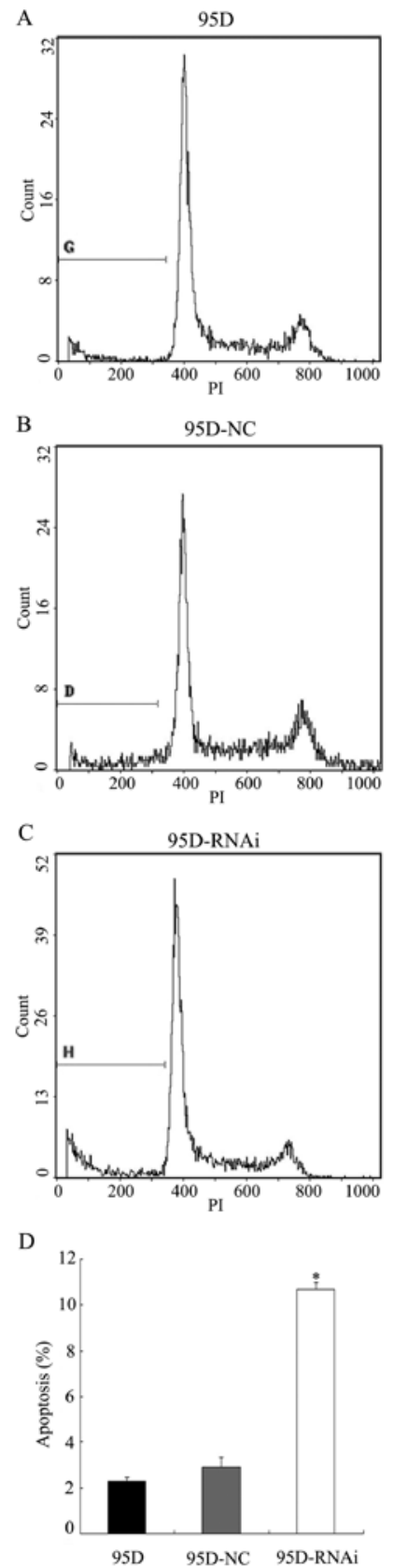

Figure 2. Knockdown of LCMR1 resulted in an increase in the percentage of apoptotic cells. (A-C) Typical examples of flow cytometric results in the 95D, 95D-NC and 95D-RNAi cell groups. (D) Comparison of the percentages of apoptotic cells in the three groups. The 95D-RNAi sample displayed a higher percentage of apoptotic cells than those in the 95D and 95D-NC cell samples. ${ }^{*} \mathrm{P}<0.05$.

cell lines: A549 (p53 wild-type cancer cells) and H1299 (p53-deficient cancer cells). A549, A549-NC, A549-RNAi, H1299, H1299-NC and H1299-RNAi cells were subjected to flow cytometric analysis $72 \mathrm{~h}$ after infection. As demonstrated in Fig. 4, the percentage of apoptotic cells in the A549RNAi group was significantly higher compared to the levels in the A549 and A549-NC groups $(\mathrm{P}<0.05)$. By contrast, the percentage of apoptotic cells in the H1299-RNAi group was not significantly different from those in the H1299 or H1299-NC group ( $>>0.05)$ (Fig. 5). These results suggest that LCMR1-mediated apoptosis is associated with the p53 pathway. 


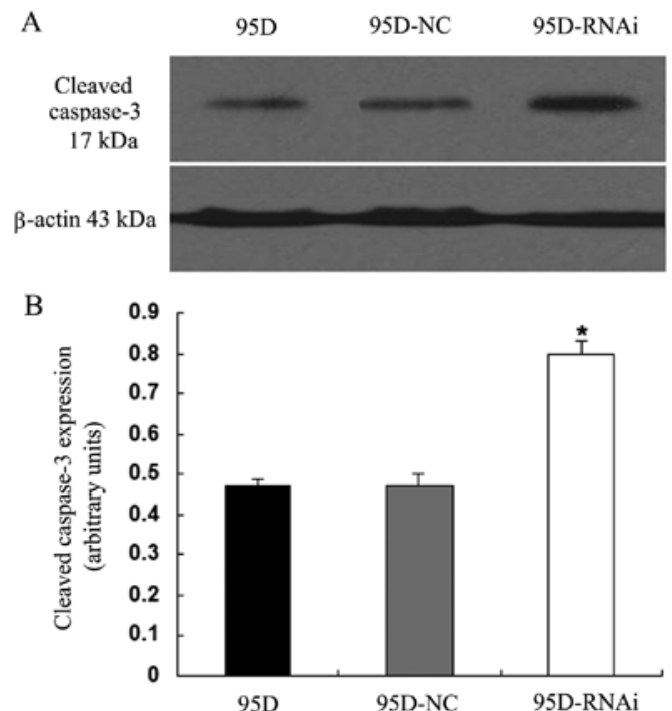

Figure 3. LCMR1 knockdown increased the expression of cleaved caspases-3. (A) An example of western blot analysis for the cleaved caspase-3 protein examined in the 95D,95D-NC and 95D-RNAi cell groups. (B) Comparison of the protein expression levels of cleaved caspase- 3 in the 3 cell groups. The expression level of this protein in the 95D-RNAi cell group was significantly higher compared to levels in the 95D and 95D-NC cell groups. $(\mathrm{P}<0.05)$.

Bax and Mcl-1 are involved in LCMR1-related cell apoptosis. p53 protein regulates the apoptotic pathway through the transcriptional activation of apoptosis-related genes, such as Bax, a pro-apoptotic gene and $\mathrm{Mcl}-1$, an anti-apoptotic gene (10). To determine whether the apoptosis induced by LCMR1 knockdown is related to Bax and Mcl-1, we examined the expression levels of these two genes in the 95D, 95D-NC and 95D-RNAi cell groups using quantitative real-time RT-PCR and western blot analyses. Bax expression was upregulated at both the mRNA and protein levels in the 95D-RNAi cells compared to levels in the 95D and 95D-NC cells $(\mathrm{P}<0.05)$ (Fig. 6). By contrast, Mcl-1 expression was downregulated at both the mRNA and protein levels in the 95D-RNAi cells $(\mathrm{P}<0.05)$ (Fig. 7). These results suggest that downregulation of LCMR1 expression leads to a differential expression change in these pro- and anti-apoptotic genes.

\section{Discussion}

LCMR1 and apoptosis. Cancer has a number of 'mission critical' events that propel tumor cells into uncontrolled expansion and invasion (11). The balance of proliferation and apoptosis plays an important role in the control of tumor growth (12). LCMR1 is a novel lung cancer-related gene that has been implicated in cell proliferation. However, the function of LCMR1 in the regulation of the apoptotic process remains unclear. In this study, we employed an RNAi approach to target LCMR1 in order to determine whether LCMR1 participates in the regulation of lung cancer cell apoptosis. Quantitative real-time RT-PCR and western blot analysis revealed that LCMR1 was successfully knocked down by RNAi at both the mRNA and protein levels. Using flow cytometry, we discovered that LCMR1 knockdown enhanced apoptotic activity in lung cancer cells. Western blot
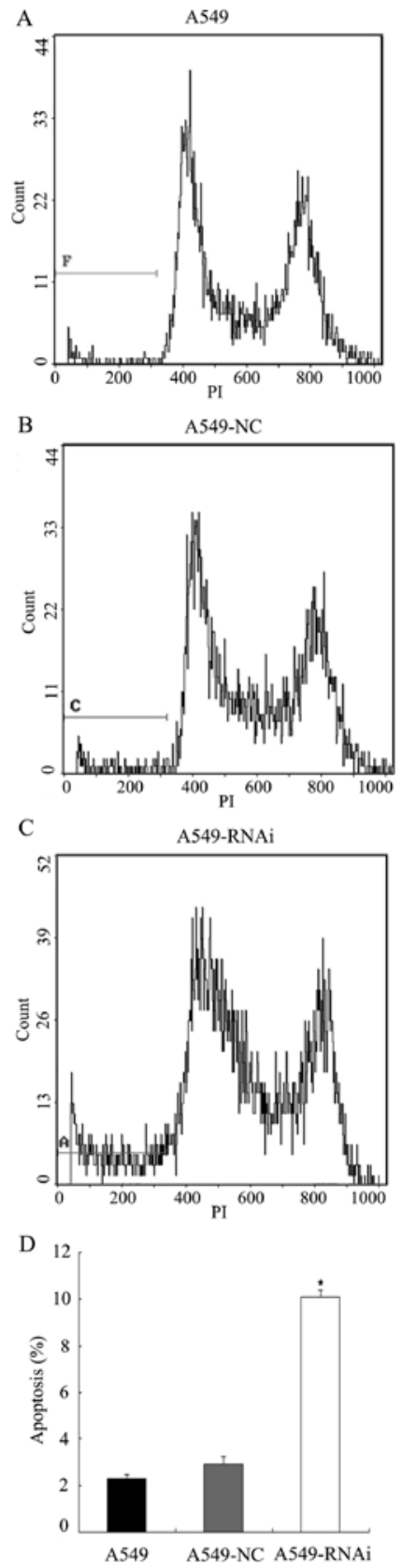

Figure 4. Suppression of LCMR1 promoted strong apoptotic activity in p53 wild-type A549 cells. (A-C) Typical examples of flow cytometric results in the A549, A549-NC and A549-RNAi cell groups. (D) Comparison of the percentages of apoptotic cells in the 3 groups. The A549-RNAi sample depicted a higher percentage of apoptotic cells compared to the A549 and A549-NC cell samples. ${ }^{*} \mathrm{P}<0.05$.

analysis demonstrated that LCMR1 knockdown increased the expression of cleaved caspase-3, an effector caspase activated in approximately all apoptotic cells. These findings suggest that LCMR1 regulates apoptotic activity in lung cancer cells, possibly acting as an apoptosis suppressor.

p53 and LCMR1-related apoptosis. p53 is a tumor suppressor gene which mediates apoptotic cell death in a variety of cell types (13-16). Lung cancer cell lines, A549 (p53 $\left.{ }^{+/+}\right)$and H1299 $\left(\mathrm{p} 53^{-/}\right)$, are widely used in the study of $\mathrm{p} 53$. In the current study, we applied these two cell lines to determine the role of p53 in LCMR1-related apoptosis. Our results demonstrated 
A
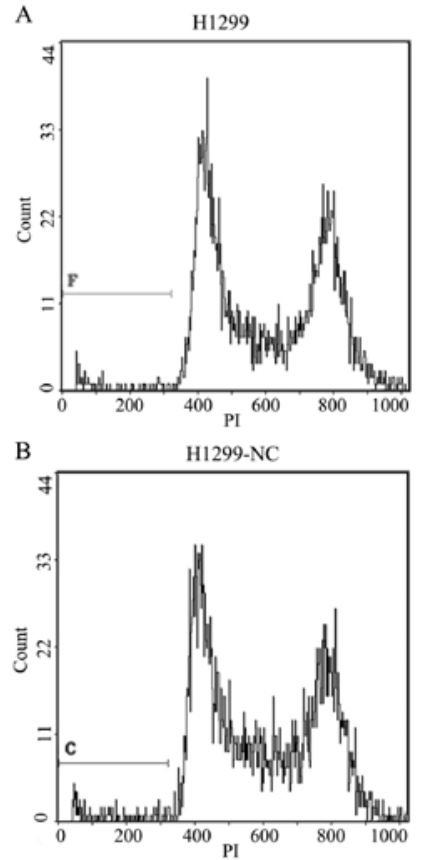

$\mathrm{C}$

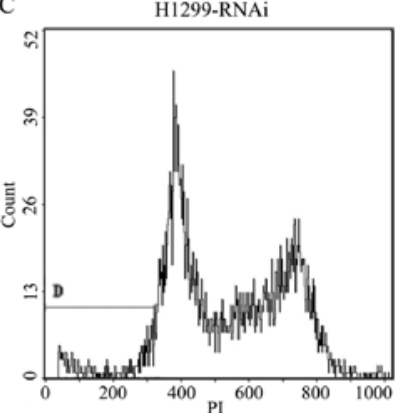

D

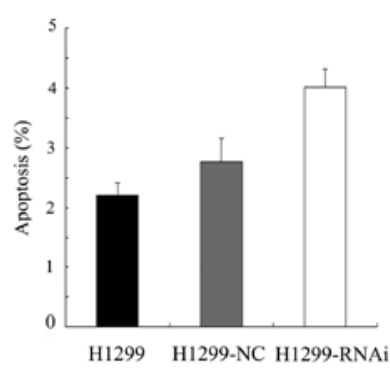

Figure 5. Suppression of LCMR1 promoted mild apoptosis in p53-deficient H1299 cells. (A-C) Typical examples of flow cytometric results in the H1299, H1299-NC and H1299-RNAi cell groups. (D) Comparison of the percentages of apoptotic cells in the three groups. The percentage of apoptotic cells in the H1299-RNAi sample was not significantly different from those in the H1299 or H1299-NC cell sample.

that the suppression of LCMR1 promoted strong apoptotic activity in p53 wild-type A549 cells, but only mild apoptosis in p53-deficient H1299 cells. These results suggest that LCMR1-related apoptosis requires the involvement of p53.

Bax and Mcl-1 in LCMR1-related apoptosis. The finding that LCMR1-mediated apoptosis is associated with the p53 pathway prompted us to explore how p53 affects LCMR1related apoptosis. p53 may exert its functions by causing the direct transcriptional induction of specific target genes such as the B-cell lymphoma 2 (Bcl-2) family. The Bcl-2 family members are central regulators of the intracellular apoptotic
A
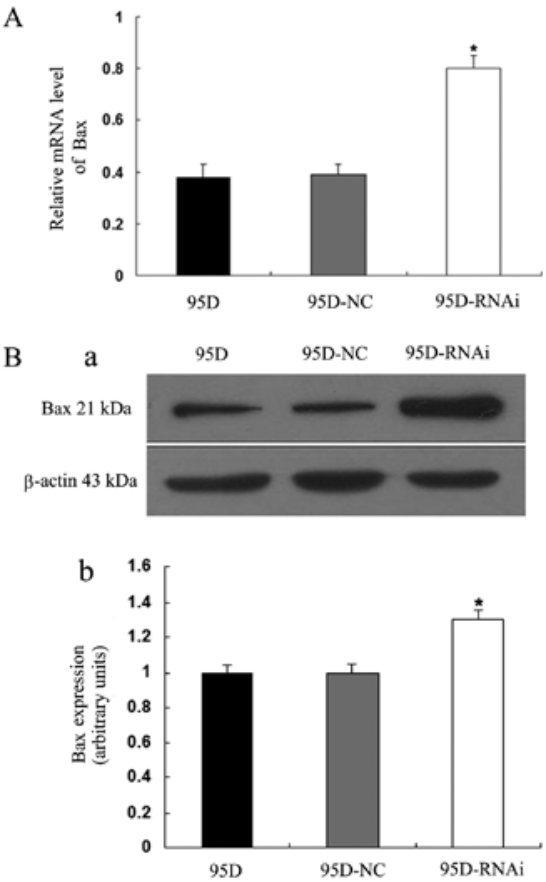

Figure 6. LCMR1 knockdown led to an increase in Bax expression. (A) Comparison of the transcriptional expression levels of Bax in the 95D, 95D-NC and 95D-RNAi cell groups. (B) a, An example of western blot analysis for the Bax protein examined in the three groups. b, Comparison of the protein expression levels of Bax in the three groups. Bax expression was upregulated at both the mRNA and protein levels in 95D-RNAi cells as compared with levels in the 95D and 95D-NC cells. ${ }^{*} \mathrm{P}<0.05$.
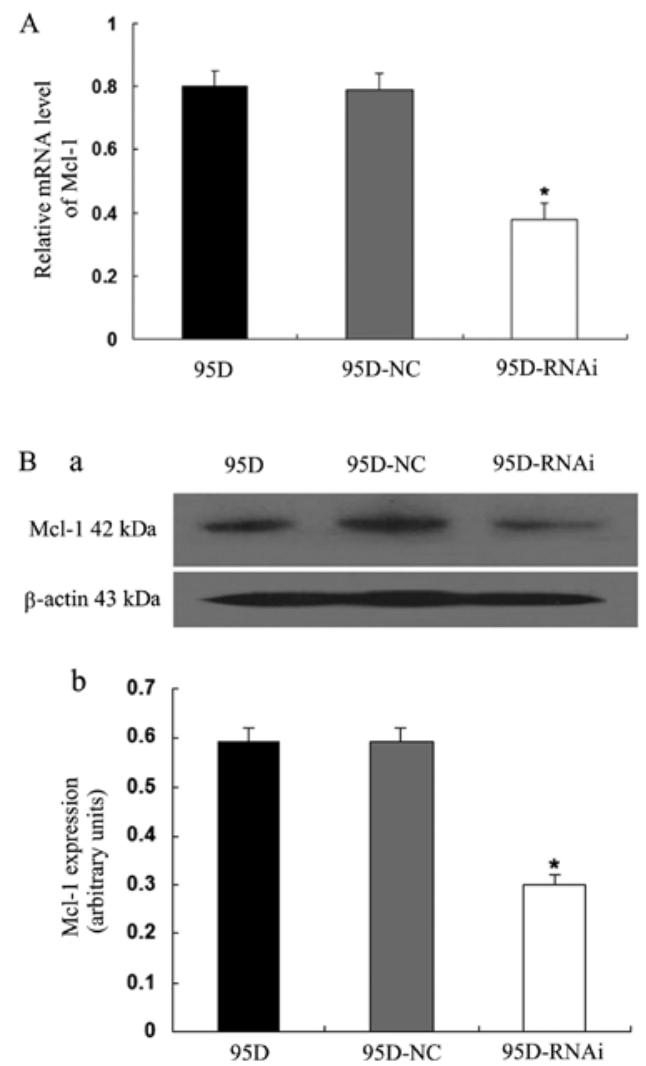

Figure 7. LCMR1 knockdown led to a decrease in Mcl-1 expression. (A) Comparison of the transcriptional expression levels of Mcl-1 in the 95D, 95D-NC and 95D-RNAi cell groups. (B) a, An example of western blot analysis for the Mcl-1 protein examined in the 3 groups. $b$, Comparison of the protein expression levels of Mcl-1 in the 3 groups. Mcl-1 expression was downregulated at both the mRNA and protein levels in the 95D-RNAi cells. ${ }^{*} \mathrm{P}<0.05$. 
signaling cascades (17). Bax (a pro-apoptotic gene) and its suppressor Mcl-1 are members of the Bcl-2 family (18). p53 directly interacts with Bax to promote Bax oligomerization (19). Oligomeric Bax induces the release of cytochrome $c$ from mitochondria, which in turn causes the activation of caspases, ultimately leading to apoptosis (20). Mcl-1 blocks apoptosis by binding and sequestering the pro-apoptotic protein Bax (21). Our results revealed that the downregulation of LCMR1 led to an increase in Bax expression and a decrease in Mcl-1 expression, suggesting a contribution of Bax and Mcl-1 in LCMR1-related apoptosis. Bax and Mcl-1 have been shown to participate in the mitochondrial signaling pathway of apoptosis (22). The question whether LCMR1 modulates apoptosis via the same signaling apoptosis pathway requires further research.

In conclusion, to the best of our knowledge, our study for the first time reveals that LCMR1 suppresses apoptosis in lung cancer cells. This effect is mediated via the p53 pathway and is associated with apoptosis-related proteins Bax and Mcl-1. Hence, this study indicates that LCMR1 may serve as a potential molecular target for lung cancer therapy.

\section{Acknowledgements}

We thank Dr Bohua $\mathrm{Hu}$ for his comments and editorial assistance. This study was supported by the National Natural Science Foundation of China (no. 30370616).

\section{References}

1. Jemal A, Siegel R, Ward E, Hao Y, Xu J, Murray T and Thun MJ: Cancer statistics, 2008. CA Cancer J Clin 58: 71-96, 2008.

2. Sharma SV, Bell DW, Settleman J and Haber DA: Epidermal growth factor receptor mutations in lung cancer. Nat Rev Cancer 7: 169-181, 2007.

3. Santarius T, Shipley J, Brewer D, Stratton MR and Cooper CS: A census of amplified and overexpressed human cancer genes. Nat Rev Cancer 10: 59-64, 2010.

4. Chen L, Liang Z, Tian Q, et al: Overexpression of LCMR1 is significantly associated with clinical stage in human NSCLC. J Exp Clin Cancer Res 30: 18, 2011.
5. Sato S, Tomomori-Sato C, Parmely TJ, et al: A set of consensus mammalian mediator subunits identified by multidimensional protein identification technology. Mol Cell 14: 685-691, 2004.

6. Ji-Fu E, Xing JJ, Hao LQ and Fu CG: Suppression of lung cancer metastasis-related protein 1 (LCMR1) inhibits the growth of colorectal cancer cells. Mol Biol Rep 39: 3675-3681, 2012.

7. Sun M, Jiang R, Li JD, et al: MED19 promotes proliferation and tumorigenesis of lung cancer. Mol Cell Biochem 355: 27-33, 2011.

8. Zou SW, Ai KX, Wang ZG, Yuan Z, Yan J and Zheng Q: The role of Med19 in the proliferation and tumorigenesis of human hepatocellular carcinoma cells. Acta Pharmacol Sin 32: 354-360, 2011.

9. Liebermann DA, Hoffman B and Steinman RA: Molecular controls of growth arrest and apoptosis: p53-dependent and independent pathways. Oncogene 11: 199-210, 1995.

10. Basu A and Haldar S: The relationship between BcI2, Bax and p53: consequences for cell cycle progression and cell death. Mol Hum Reprod 4: 1099-1109, 1998.

11. Evan GI and Vousden KH: Proliferation, cell cycle and apoptosis in cancer. Nature 411: 342-348, 2001.

12. Mattern $\mathbf{J}$ and Volm M: Imbalance of cell proliferation and apoptosis during progression of lung carcinomas. Anticancer Res 24: 4243-4246, 2004.

13. Cong WM, Bakker A, Swalsky PA, et al: Multiple genetic alterations involved in the tumorigenesis of human cholangiocarcinoma: a molecular genetic and clinicopathological study. J Cancer Res Clin Oncol 127: 187-192, 2001.

14. Gottlieb TM and Oren M: p53 and apoptosis. Semin Cancer Biol 8: 359-368, 1998.

15. Amaral JD, Xavier JM, Steer CJ and Rodrigues CM: The role of p53 in apoptosis. Discov Med 9: 145-152, 2010.

16. Fridman JS and Lowe SW: Control of apoptosis by p53. Oncogene 22: 9030-9040, 2003.

17. Danial NN: BCL-2 family proteins: critical checkpoints of apoptotic cell death. Clin Cancer Res 13: 7254-7263, 2007.

18. Craig RW: The bcl-2 gene family. Semin Cancer Biol 6: 35-43, 1995.

19. Chipuk JE, Kuwana T, Bouchier-Hayes L, Droin NM, Newmeyer DD, Schuler M and Green DR: Direct activation of Bax by 553 mediates mitochondrial membrane permeabilization and apoptosis. Science 303: 1010-1014, 2004.

20. Antonsson B, Montessuit S, Lauper S, Eskes R and Martinou JC: Bax oligomerization is required for channel-forming activity in liposomes and to trigger cytochrome $\mathrm{c}$ release from mitochondria. Biochem J 345: 271-278, 2000.

21. Thomas LW, Lam C and Edwards SW: Mcl-1; the molecular regulation of protein function. FEBS Lett 584: 2981-2989, 2010.

22. Someya S, Xu J, Kondo K, et al: Age-related hearing loss in C57BL/6J mice is mediated by Bak-dependent mitochondrial apoptosis. Proc Natl Acad Sci USA 106: 19432-19437, 2009. 